\title{
Prevalence of non-alcoholic fatty liver disease on computed tomography in patients with inflammatory bowel disease visiting an emergency department
}

\author{
Alisa Likhitsuppa, , Jason Dundulis ${ }^{a}$, Shaya Ansaric, Hani El-Halawanya , Randal Michelsona, \\ Colleen Hutton ${ }^{d}$, Kevin Kennedy ${ }^{\mathrm{e}}$, John H. Helzberg ${ }^{\mathrm{a}, \mathrm{b}}$, Rajiv Chhabra ${ }^{\mathrm{a}, \mathrm{b}}$ \\ University of Missouri Kansas City; Saint Luke's Hospital of Kansas City; Mid-America Heart Institute St. Luke's Health \\ System, Kansas City, MO, USA
}

\begin{abstract}
Background Non-alcoholic fatty liver disease (NAFLD) is common in patients with inflammatory bowel disease (IBD). The purpose of this study was to further examine the prevalence of NAFLD in IBD patients.

Methods We retrospectively reviewed the medical records of IBD patients who visited the emergency department because of abdominal pain between January 2009 and December 2014. These were compared with a group of 70 controls without IBD, matched for age and body mass index (BMI). Computed tomography data were analyzed for the presence or absence of hepatic steatosis. Patient with recent steroid or excessive alcohol use were excluded. Univariate and multivariate analyses were performed.
\end{abstract}

Results NAFLD prevalence was $44 \%(31 / 70)$ in the IBD group vs. $16 \%$ (11/70) in controls $(\mathrm{P}<0.001)$. There was no significant difference between the 2 groups in age, sex distribution, BMI, presence of diabetes, or levels of serum transaminases, serum albumin or platelets. In multivariate analysis, the presence of IBD was independently associated with NAFLD (odds ratio 4.53, 95\% confidence interval 2.00-10.26; $\mathrm{P}=0.002$ ).

Conclusions The presence of IBD is strongly and independently associated with NAFLD. Systemic inflammation and alteration of the intestinal microbiome have been proposed as mechanisms, but further studies are needed to better elucidate the pathophysiology.

Keywords Hepatic steatosis, inflammatory bowel disease, non-alcoholic fatty liver disease

Ann Gastroenterol 2019; 32 (3): 1-4

Department of a Gastroenterology and Hepatology, University of Missouri Kansas City (Alisa Likhitsup, Jason Dundulis, Hani El-Halawany, Randal Michelson, John H. Helzberg, Rajiv Chhabra); 'Gastroenterology and Hepatology, Saint Luke's Hospital of Kansas City (Alisa Likhitsup, John H. Helzberg, Rajiv Chhabra); 'Radiology, University of Missouri Kansas City (Shaya Ansari); ${ }^{\mathrm{d} C l i n i c a l}$ Research, Mid-America Heart Institute St. Luke's Health System (Colleen Hutton); 'Biostatistics, Mid-America Heart Institute St. Luke's Health System (Kevin Kennedy), Kansas City, MO, USA

Conflict of Interest: None

Correspondence to: Alisa Likhitsup, MD, 4320 Wornall Rd, Suit 240, Kansas City, MO 64111, USA, e-mail: alikhitsup@gmail.com

Received 6 January 2019; accepted 1 February 2019; published online 15 March 2019

DOI: https://doi.org/10.20524/aog.2019.0371

\section{Introduction}

Non-alcoholic fatty liver disease (NAFLD) is more common in the western world, with a prevalence of $19-46 \%$ in the general US population [1-13]. Risk factors for NAFLD include obesity, insulin resistance, diabetes mellitus (DM), hypertension, and hypertriglyceridemia [14]. NAFLD in patients with inflammatory bowel disease (IBD) has become increasingly recognized, with the prevalence of NAFLD in IBD patients ranging from $8-59 \%$ [15-19]. The pathogenesis of NAFLD among the IBD population is not well understood. Other than metabolic syndrome, IBD-specific risk factors, including malnutrition, intestinal inflammation, alterations of gut microbiota, steroid exposure, and drug-induced hepatotoxicity may also contribute to pathogenesis of NAFLD [20].

The aim of this study was to evaluate the prevalence of NAFLD in IBD patients compared with a matched controlled group and to identify the characteristics associated with NAFLD in IBD patients. 


\section{Patients and methods}

The Institutional Review Board of Saint Luke's Hospital of Kansas City approved the data collection and methodology for this study. We conducted a retrospective study of IBD patients 18 years of age or older who presented to the emergency department (ED) with a chief complaint of abdominal pain and who completed an abdominal computed tomography (CT) scan in their evaluation. ED encounters within the medical records database between 2009 and 2014 were queried for ICD-9 codes associated with abdominal pain and IBD (either Crohn's disease or ulcerative colitis). The search was further refined to include only encounters in which abdominal CT scans were performed, based on billing codes associated with the visits. ED provider notes and, when applicable, admission notes were reviewed for possible exclusion criteria, including significant alcohol use, defined as $>7$ drinks per week in females and $>14$ drinks per week in males, known liver disease other than NAFLD, and use of corticosteroids within the preceding 12 months. A control group, matched by age ( \pm 3 years) and body mass index (BMI; \pm 2.5 ), was compiled from patients without IBD who presented to the ED for abdominal pain over the same time period and underwent abdominal CT scans. Medical records were reviewed and the following data were collected: age, BMI, presence of DM, IBD type, levels of aspartate aminotransferase (AST), alanine aminotransferase (ALT), total bilirubin, alkaline phosphatase, albumin, and platelet count. The CT scans of all patients were reviewed specifically for the presence of NAFLD by a specialized abdominal radiologist (SA). Patients were determined to have NAFLD if the liver parenchyma demonstrated a lower density compared with the spleen of $>10$ Hounsfield units (HU). The primary endpoint was the presence or absence of hepatic steatosis based on CT imaging.

\section{Statistical analysis}

The data were presented as mean \pm standard deviation for continuous variables and as a total percentage for categorical variables. Student's $t$-test and chi-square tests were used in the unadjusted analysis. The predictive factors for NAFLD were assessed with a multivariate logistic regression model adjusted for age, sex, BMI, the presence of DM and transaminase levels. SPSS version 21.0 (IBM Corp, Armonk, NY) was used for all analyses.

\section{Results}

Of the 70 patients with IBD, 31 (44\%) had hepatic steatosis on abdominal CT images, compared with only $16 \%$ (11 of 70 ) of controls. The difference in NAFLD prevalence between the 2 groups was statistically significant $(\mathrm{P}<0.001)$. Patient characteristics, including age, sex, $\mathrm{BMI}$, and the presence of diabetes were similar across the 2 groups (Table 1). There were no significant differences between the 2 groups in transaminase or albumin levels or platelet count (Table 2). The predictive factors associated with NAFLD were examined in a multivariate logistic regression model adjusted for age, sex, BMI, presence of DM, and transaminase levels. The presence of IBD was independently associated with NAFLD, with an odds ratio of 4.53 (95\% confidence interval [CI] 2.00-10.26, $\mathrm{P}=0.002$ ) (Fig. 1).

Table 1 Characteristics of patients enrolled in the cohort

\begin{tabular}{|c|c|c|c|c|}
\hline Characteristics & $\begin{array}{c}\text { Total } \\
(\mathrm{n}=140)\end{array}$ & $\begin{array}{c}\text { IBD } \\
(\mathrm{n}=70)\end{array}$ & $\begin{array}{c}\text { Control }^{*} \\
(\mathrm{n}=70)\end{array}$ & $\mathrm{P}$-value \\
\hline Age & $38.7 \pm 14.9$ & $38.6 \pm 15$ & $38.8 \pm 15$ & 0.95 \\
\hline Male sex & $58(41 \%)$ & $31(44 \%)$ & $27(39 \%)$ & 0.49 \\
\hline BMI & $26.7 \pm 6.7$ & $26.6 \pm 6.9$ & $26.7 \pm 6.6$ & 0.65 \\
\hline Crohn's disease & $57(81 \%)$ & $57(81 \%)$ & N/A & \\
\hline Diabetes & $14(10 \%)$ & $8(11 \%)$ & $6(9 \%)$ & 0.57 \\
\hline NAFLD & $42(30 \%)$ & $31(44 \%)$ & $11(16 \%)$ & $<0.001^{\dagger}$ \\
\hline
\end{tabular}

${ }^{*}$ Age- and BMI-matched control group

Continuous variables were compared using Student's t-test. Category variables were compared using the chi-square or Fisher's exact test

${ }^{\dagger}$ Alpha less than 0.05 considered statistically significant

$B M I$, body mass index; IBD, inflammatory bowel disease; NAFLD, non alcoholic fatty liver disease; N/A, not applicable

Table 2 Laboratory data of patients enrolled in the cohort

\begin{tabular}{lcccc}
\hline Lab data & $\begin{array}{c}\text { Total } \\
(\mathrm{n}=140)\end{array}$ & $\begin{array}{c}\text { IBD } \\
(\mathrm{n}=70)\end{array}$ & $\begin{array}{c}\text { Control }^{*} \\
(\mathrm{n}=70)\end{array}$ & P-value \\
\hline AST & $35 \pm 38$ & $32 \pm 39$ & $38 \pm 36$ & 0.7 \\
ALT & $38 \pm 56$ & $34 \pm 34$ & $42 \pm 71$ & 0.19 \\
Albumin & $4 \pm 0.6$ & $3.8 \pm 0.6$ & $4.3 \pm 0.5$ & 0.24 \\
Platelet count & $267 \pm 93$ & $286 \pm 101$ & $250 \pm 82$ & 0.07 \\
\hline
\end{tabular}

${ }^{*}$ Age- and BMI-matched control group

Continuous variable compared using Student's t-test. Category variables compared using chi-square or Fisher's exact test

BMI, body mass index; ALT, alanine aminotransferase; AST, aspartate aminotransferase; IBD, inflammatory bowel disease

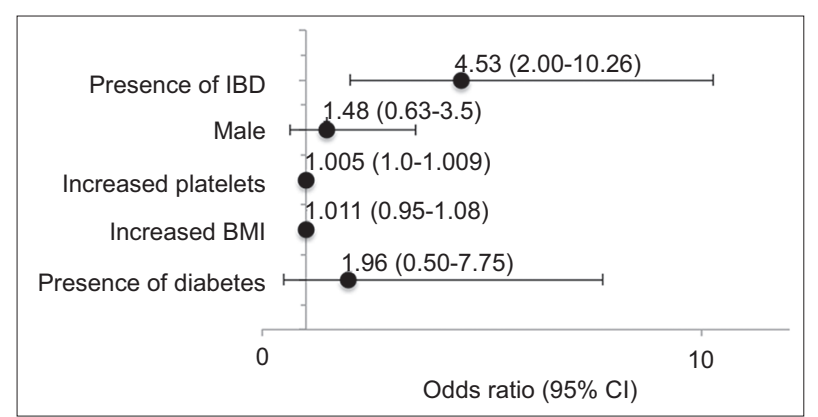

Figure 1 Predictive factors associated with the presence of NAFLD from the study cohort, adjusted for age, male sex, BMI, presence of diabetes, and transaminase levels $(n=140)$

NAFLD, non-alcoholic fatty liver disease; IBD, inflammatory bowel disease; BMI, body mass index; CI, confidence interval 


\section{Discussion}

Because of the obesity epidemic and the increasing prevalence of metabolic syndrome, the global prevalence of NAFLD has been rising and is now estimated to be $24 \%$ [13]. The reported prevalence of NAFLD in IBD patients has varied from $8-59 \%$, depending on the diagnostic criteria: $59 \%$ based on liver histology, $34 \%$ based on hepatic steatosis index score, $8 \%$ based on combined multiple imaging modalities, and $26-40 \%$ based on ultrasonography [15-19]. In this population, NAFLD was diagnosed in $44 \%$ of IBD patients on the basis of abdominal CT results, compared with $16 \%$ in non-IBD patients. The diagnostic value of abdominal CT for NAFLD has been investigated and a liver-to-spleen difference in attenuation of $>9$ HU had a specificity of $100 \%$ and a sensitivity of $82 \%$ in the detection of moderate to severe hepatic steatosis [20]. In our study, patients were determined to have NAFLD if the liver-to-spleen difference in attenuation was $>10 \mathrm{HU}$. Hypertension, obesity and metabolic syndrome were associated with the presence of NAFLD in IBD patients $[16,19]$. We compared our study population of IBD patients with an age- and BMI-matched controlled group; there were no significant differences in metabolic profile between the 2 groups.

Of our IBD study population, $81 \%$ were diagnosed with Crohn's disease, whereas 19\% had ulcerative colitis. The sample sizes were too small to perform a subgroup analysis to identify the distinction between Crohn's disease and ulcerative colitis in relation to NAFLD prevalence.

Some studies have reported that IBD disease activity or severity and prior small bowel surgery were associated with NAFLD [17,21] However, other studies reported no such association between NAFLD and IBD [19,22]. In this study group, the presence of IBD was very strongly associated with NAFLD, with an odds ratio of 4.53 (95\%CI 2.00-10.26), $\mathrm{P}=0.002$.

A limitation of this study was the incomplete data recording of our ED cohort. A detailed smoking history, IBD disease severity, duration of diagnosis, prior IBDrelated surgery, and IBD medications or treatment were not completely noted. As the study evaluated an ED population, there may have been an inherent selection bias towards patients with more severe disease activity and less medical compliance. Since glucocorticoids have been implicated in the pathogenesis of NAFLD, we excluded patients who had taken corticosteroids within the previous 12 months. Thus, the NAFLD prevalence reported in our study does not apply to IBD patients exposed to corticosteroid therapy within a 1-year period.

In conclusion, data concerning the association between IBD disease activity and NAFLD are conflicting. Our results from this controlled study demonstrate that the presence of IBD in patients who visited the ED was strongly associated with NAFLD. Systemic inflammation and alteration of the intestinal microbiome have been proposed as potential causative factors, but further studies will be needed to elucidate the pathophysiology.

\section{Summary Box}

\section{What is already known:}

- The global prevalence of non-alcoholic fatty liver disease (NAFLD) has been rising because of the epidemic of obesity and metabolic syndrome

- The prevalence of NAFLD in patients with inflammatory bowel disease (IBD) reportedly varies from $8-59 \%$, depending on the diagnostic criteria

- Hypertension, obesity and metabolic syndrome are associated with NAFLD in IBD patients

- Data regarding IBD disease severity and prior small bowel surgery in association with NAFLD in IBD patients are conflicting

\section{What the new findings are:}

- Based on abdominal computed tomography, NAFLD was also more common (44\%) in IBD patients who visited the emergency department, compared with a control group without IBD

- NAFLD is independently associated with the presence of IBD

- Further studies are still needed to elucidate the pathophysiology of NAFLD development in IBD populations

\section{References}

1. Setji TL, Holland ND, Sanders LL, Pereira KC, Diehl AM, Brown AJ. Nonalcoholic steatohepatitis and nonalcoholic fatty liver disease in young women with polycystic ovary syndrome. J Clin Endocrinol Metab 2006;91:1741-1747.

2. Mohanty SR, Troy TN, Huo D, O’Brien BL, Jensen DM, Hart J. Influence of ethnicity on histological differences in non-alcoholic fatty liver disease. J Hepatol 2009;50:797-804.

3. Lazo M, Hernaez R, Bonekamp S, et al. Non-alcoholic fatty liver disease and mortality among US adults: prospective cohort study. BMJ 2011;343:d6891.

4. Williams CD, Stengel J, Asike MI, et al. Prevalence of nonalcoholic fatty liver disease and nonalcoholic steatohepatitis among a largely middle-aged population utilizing ultrasound and liver biopsy: a prospective study. Gastroenterology 2011;140:124-131.

5. Younossi ZM, Stepanova M, Negro F, et al. Nonalcoholic fatty liver disease in lean individuals in the United States. Medicine (Baltimore) 2012;91:319-327.

6. Kim D, Kim WR, Kim HJ, Therneau TM. Association between noninvasive fibrosis markers and mortality among adults with nonalcoholic fatty liver disease in the United States. Hepatology 2013;57:1357-1365.

7. Lazo M, Hernaez R, Eberhardt MS, et al. Prevalence of nonalcoholic fatty liver disease in the United States: the Third National Health and Nutrition Examination Survey, 1988-1994. Am J Epidemiol 2013;178:38-45.

8. Ruhl CE, Everhart JE. Relationship of non-alcoholic fatty liver 
disease with cholecystectomy in the US population. Am J Gastroenterol 2013;108:952-958.

9. Otgonsuren M, Stepanova M, Gerber L, Younossi ZM. Anthropometric and clinical factors associated with mortality in subjects with nonalcoholic fatty liver disease. Dig Dis Sci 2013; 58:1132-1140.

10. Younossi ZM, Otgonsuren M, Venkatesan C, Mishra A. In patients with non-alcoholic fatty liver disease, metabolically abnormal individuals are at a higher risk for mortality while metabolically normal individuals are not. Metabolism 2013;62:352-360.

11. Smits MM, Ioannou GN, Boyko EJ, Utzschneider KM. Nonalcoholic fatty liver disease as an independent manifestation of the metabolic syndrome: results of a US national survey in three ethnic groups. J Gastroenterol Hepatol 2013;28:664-670.

12. Schneider AL, Lazo M, Selvin E, Clark JM. Racial differences in nonalcoholic fatty liver disease in the U.S. population. Obesity (Silver Spring) 2014;22:292-299.

13. Younossi ZM, Koenig AB, Abdelatif D, Fazel Y, Henry L, Wymer M. Global epidemiology of nonalcoholic fatty liver diseasemeta-analytic assessment of prevalence, incidence, and outcomes. Hepatology 2016;64:73-84.

14. Chalasani N, Younossi Z, Lavine JE, et al. The diagnosis and management of nonalcoholic fatty liver disease: Practice guidance from the American Association for the Study of Liver Diseases. Hepatology 2018;67:328-357.

15. Bargiggia S, Maconi G, Elli M, et al. Sonographic prevalence of liver steatosis and biliary tract stones in patients with inflammatory bowel disease: study of 511 subjects at a single center. J Clin Gastroenterol 2003;36:417-420.

16. Sourianarayanane A, Garg G, Smith TH, Butt MI, McCullough AJ, Shen B. Risk factors of non-alcoholic fatty liver disease in patients with inflammatory bowel disease. JCrohns Colitis 2013;7:e279-e285.

17. Bessissow T, Le NH, Rollet K, Afif W, Bitton A, Sebastiani G. Incidence and predictors of nonalcoholic fatty liver disease by serum biomarkers in patients with inflammatory bowel disease. Inflamm Bowel Dis 2016;22:1937-1944.

18. Bosch DE, Yeh MM. Primary sclerosing cholangitis is protective against nonalcoholic fatty liver disease in inflammatory bowel disease. Hum Pathol 2017;69:55-62.

19. Carr RM, Patel A, Bownik H, et al. Intestinal inflammation does not predict nonalcoholic fatty liver disease severity in inflammatory bowel disease patients. Dig Dis Sci 2017;62:1354-1361.

20. Park SH, Kim PN, Kim KW, et al. Macrovesicular hepatic steatosis in living liver donors: use of CT for quantitative and qualitative assessment. Radiology 2006;239:105-112.

21. Jamali R, Biglari M, Seyyed Hosseini SV, Shakouri Rad A, Kosari F. The correlation between liver fat content and ulcerative colitis disease severity. Acta Med Iran 2017;55:333-339.

22. Principi M, Iannone A, Losurdo G, et al. Nonalcoholic fatty liver disease in inflammatory bowel disease: prevalence and risk factors. Inflamm Bowel Dis 2018;24:1589-1596. 